\title{
How to adjust endoscopic findings to histopathological findings of the stomach: a "histopathology-oriented" correspondence method helps to understand endoscopic findings
}

\author{
Yasuko Fujita $^{1,2}$ (1) Mitsuo Kishimoto ${ }^{3} \cdot$ Osamu Dohi $^{4} \cdot$ Kazuhiro Kamada $^{4} \cdot$ Atsushi Majima $^{4}$. \\ Reiko Kimura-Tsuchiya $^{4,5}$ - Nobuaki Yagi ${ }^{4,6} \cdot$ Hideyuki Konishi $^{4} \cdot$ Yuji Naito $^{4} \cdot$ Yoshinori Harada $^{2} \cdot$ Hideo Tanaka $^{2}$. \\ Eiichi Konishi $^{3} \cdot$ Tamotsu Sugai $^{1} \cdot$ Akio Yanagisawa $^{3,7}$
}

Received: 29 May 2017 / Accepted: 23 August 2017 / Published online: 5 September 2017

(C) The International Gastric Cancer Association and The Japanese Gastric Cancer Association 2017

\begin{abstract}
Background Endoscopic findings have now become nearly as detailed as histopathological findings. Thus, one-to-one correspondence and precise feedback of histopathological findings is very desirable but may be very difficult to accomplish. We describe a systematic process called the Kyoto One-to-One Correspondence Method (the KOTO Method) that allows detailed adjustments of endoscopic findings to match histopathological findings.

Methods and results By comparing endoscopic and stereoscopic images of the gastric mucosa, we could obtain one-to-one correspondence between endoscopic images and equivalent histology in 44 of 47 fields.

Conclusions The histological structure of gastric cancers of the same histological subtype may not be similar. One-toone correspondence between endoscopic images and gastric
\end{abstract}

Yasuko Fujita

fujitaya@iwate-med.ac.jp

1 Department of Molecular Diagnostic Pathology, School of Medicine, Iwate Medical University, 19-1 Uchimaru, Morioka 020-0023, Japan

2 Department of Pathology and Cell Regulation, Kyoto Prefectural University of Medicine, Kyoto, Japan

3 Department of Surgical Pathology, Kyoto Prefectural University of Medicine, Kyoto, Japan

4 Department of Molecular Gastroenterology and Hepatology, Kyoto Prefectural University of Medicine, Kyoto, Japan

5 Department of Medical Oncology, Fukushima Medical University, Fukushima, Japan

6 Department of Gastroenterology, Murakami Memorial Hospital, Asahi University, Gifu, Japan

7 Department of Pathology, Kyoto Daiichi Red Cross Hospital, Kyoto, Japan mucosal histology (histopathology-oriented correspondence) will improve endoscopic diagnosis and provide more useful information for pathological diagnosis.

Keywords Stomach $\cdot$ Endoscopy $\cdot$ Pathology $\cdot$ Gastric cancer

\section{Introduction}

We propose a systematic process to achieve detailed adjustments of endoscopic findings to fit histopathological findings, named the Kyoto One-To-One Correspondence Method (KOTO Method). The method involves histopathologyoriented correspondence that enables nearly one-to-one conformity at the glandular level. This method may lead to improvements in endoscopy and eventually in pathological diagnosis.

At present, early gastric cancers (EGCs) [1] are detected mainly by endoscopy. Many of these, those with a negligible risk of lymph node metastasis [2-4], are treated by endoscopic resection, such as endoscopic submucosal dissection (ESD) [5]. Recent advances in endoscopy have enabled obtaining precise, magnified images with high spatial resolution. Image-enhanced endoscopy (IEE) is a useful modality for diagnosing gastric neoplasms with high accuracy, sensitivity, and specificity [6-10]. Use of IEE provides discrimination at a single gland level on images and allows conjecture on the extent and histological structure of the tumor based on its surface structure. Because endoscopic findings are nearly as detailed as histopathological findings, it is necessary to clarify the precise correlation between endoscopic and histological images.

Many studies have attempted to correlate pathological images to endoscopic images. Some studies have examined 
correspondence in a small area using two markings as landmarks [11]. Yagi et al. examined the detailed relationship between endoscopic findings and histology by investigating the length of the intervening section and depth of the glands [12]. However, the variety of mucosal gland structures and the degree of inflammation has caused endoscopic diagnosis of gastric mucosal diseases to be difficult, with the correspondence between endoscopic findings and histology being unreliable. If it is possible to achieve complete one-to-one correspondence between endoscopic findings and histology at a single gland level, this would allow observing the quintessence of the endoscopic images and determining the correlation between tumor surface structure and histology, leading to increased accuracy of endoscopic diagnosis including the extent of the tumor. Advances in endoscopic diagnostic skills would also be useful for planning appropriate treatment after endoscopic detection or observation of tumors.

\section{Materials and methods}

\section{Materials}

Gastric materials of 34 cases (47 fields) obtained by ESD for EGC at the hospital of the Kyoto Prefectural University of Medicine (KPUM) were examined retrospectively. After resection, the specimens were fixed with $10 \%$ neutral buffered formalin. Only the mucosa was sectioned at 2- to 3-mm intervals, with the lower submucosal tissue not being completely removed (Fig. 1). The entire specimen material,

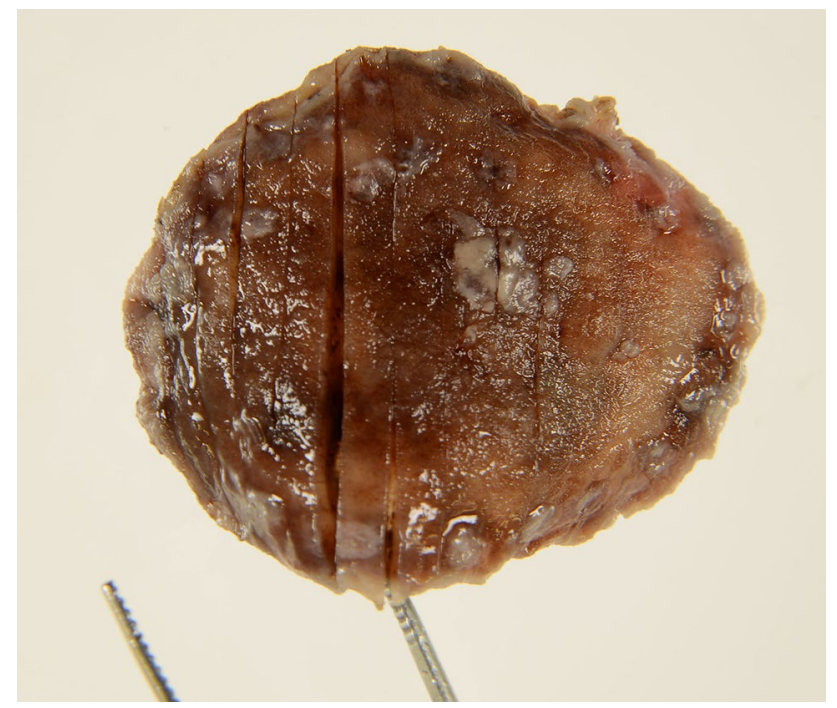

Fig. 1 Mucosal cleavage material resected by endoscopic submucosal dissection (ESD) after fixation with $10 \%$ neutral buffered formalin. Because the submucosa was not completely cut off, the cleaved mucosa was not out of position and we could recognize the continuity of the surface structure in more magnified images (Fig. 3) immersed in water, was photographed with a color digital camera (Nikon D800; Nikon Corporation, Tokyo, Japan). Each section of the material was magnified and observed by a stereomicroscope (Nikon SMZ-10; Nikon) and then photographed with a digital camera system for microscopy (Nikon DS-Fi2-L3; Nikon). After acquisition of the pictures, all the material was cut into sections. The sections were embedded in paraffin blocks, sliced into $3-\mu \mathrm{m}$ preparations, and stained with hematoxylin and eosin (H\&E) for examination and diagnosis by the pathologists (Y. Fujita, M. Kishimoto, A. Yanagisawa).

\section{Adjustment process}

We retrospectively compared the endoscopic, stereomicroscopic, and histological images using the following four-step process.

\section{Step 1: Adjusting the whole image (Fig. 2)}

Histological images scanned by the virtual slide system (NanoZoomer 2.0-HT; Hamamatsu Photonics, Hamamatsu, Japan) were saved in the JPEG format using viewer software (NDP View; Hamamatsu). The images were then processed using image processing software (Adobe Photoshop Elements 15; Adobe Systems, San Jose, CA, USA). The background white area of the JPEG-formatted histological images opened by the software was selected using the Magic Wand selection tool, with the tolerance value set to approximately 10 . The selection was then inversed by choosing

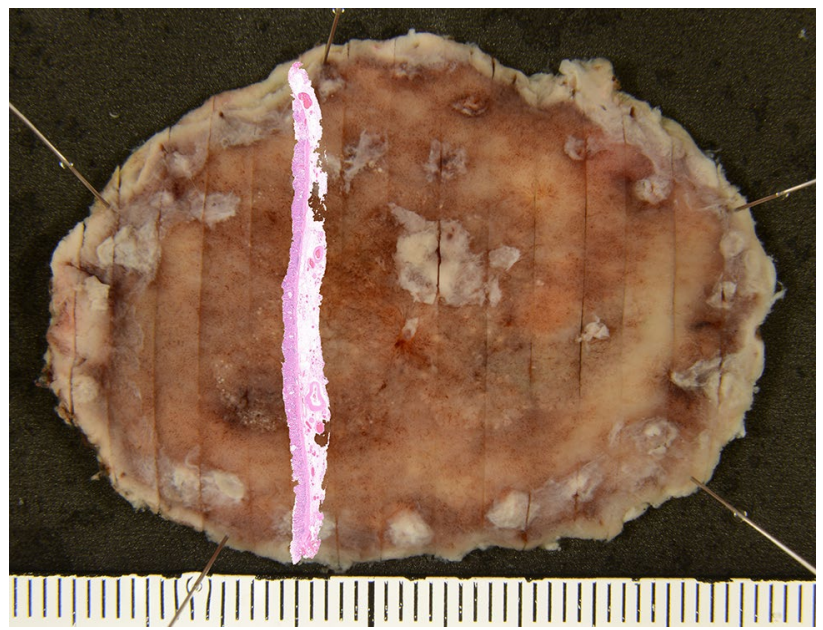

Fig. 2 Step 1: The entire picture of mucosa-cut tissue in water overlaid with the hematoxylin and eosin (H\&E) section image. Immersion of the material in water resulted in disappearance of the mucosal surface reflection, allowing clear observation of surface structures. The whole material picture overlaid with the H\&E section indicates the outline of the correspondence between surface structures and the histological image 
Select $>$ Inverse, and copied by choosing Edit $>$ Copy. The picture of the whole material or the stereomicroscopic image was also opened using the image processing software and placed on a copied histological image by choosing Edit $>$ Paste. The length of the pasted histological image was then fitted into the whole material image.

\section{Step 2: Adjusting the magnified area (Fig. 3)}

Because the histology-adjusted whole-material image showed exact correlation between surface structure and histology, we could examine each part in detail by processing more magnified stereomicroscopic and histological images in a similar manner.

\section{Step 3: Adjusting the endoscopic image and histology}

(Figs. 4, 5)

After adjusting the magnified images, we compared the stereoscopic and endoscopic images of the same area. The surface structures of the two images showed close correlation. The histological image was then adjusted to the endoscopic image with reference to the adjusted stereoscopic image as an indicator.

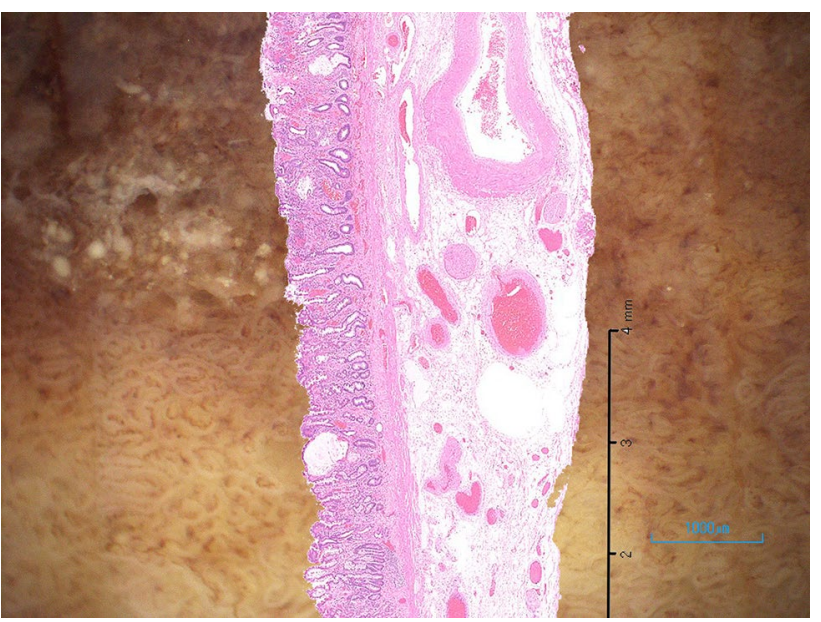

Fig. 3 Step 2: Stereoscopic image overlaid with the H\&E section image. Both images are the same magnification by making both scales the same size. By referring to the whole overlaid picture (Fig. 2), the image of the H\&E section was laid on the mucosa-cut line, and the correct line in which the H\&E section was made was determined by observing the crypt intervals or some characteristic features such as elevation or depression of mucosa and mucin accumulation as indicators. Both adjacent mucosa-cut lines are observed dimly at about $500 \mu \mathrm{m}$ from the right and left sides of this picture

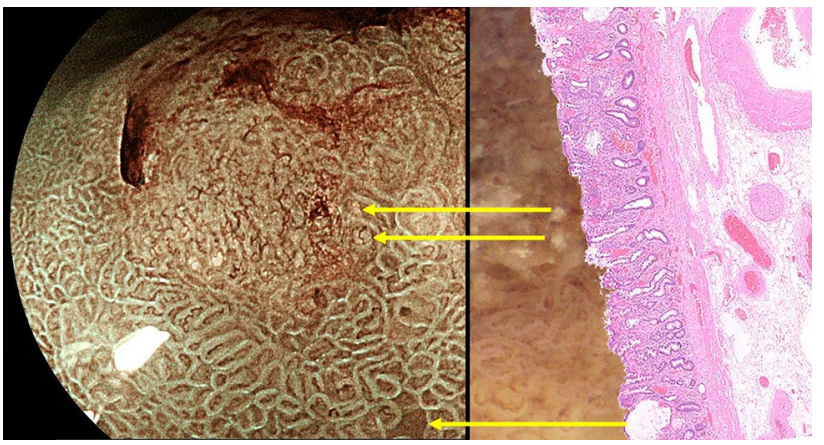

Fig. 4 Step 3: Comparison of the endoscopic image with blue laser imaging-bright mode (BLI-bright mode) to the stereoscopic image. Some characteristic features were found in both endoscopic and stereoscopic images

Step 4: Change in the opacity of the histological image (Figs. 6, 7)

When the histological image layer was selected and the opacity set to less than $100 \%$ at approximately $30-70 \%$ density, the histological overlay layer was transparent, and we could see the underlying endoscopic image dimly through the histological layer.

\section{Criterion of evaluation}

We defined successful correspondence as a field in which at least 5 points of white zone could be completely adjusted to each gland, as shown by the yellow arrows in Fig. 5 .

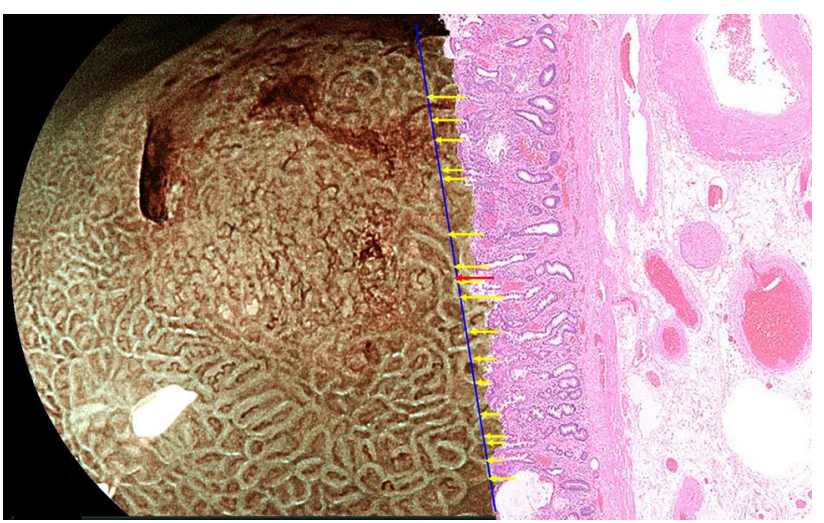

Fig. 5 Step 3 (continued): Correspondence between the endoscopic and histological images. The yellow arrows indicate correspondence of the white zone and epithelium at the edge of the gland; the red arrow indicates the demarcation line, that is, the cancer presented in the upper area from the red arrow. The blue line represents the correct line in which the $\mathrm{H} \& \mathrm{E}$ section was made. However, the $\mathrm{H} \& \mathrm{E}$ section image did not fit completely on the side (i.e., in this case, the upper and lower sides of the endoscopic picture) as the result of distortion associated with endoscopy 


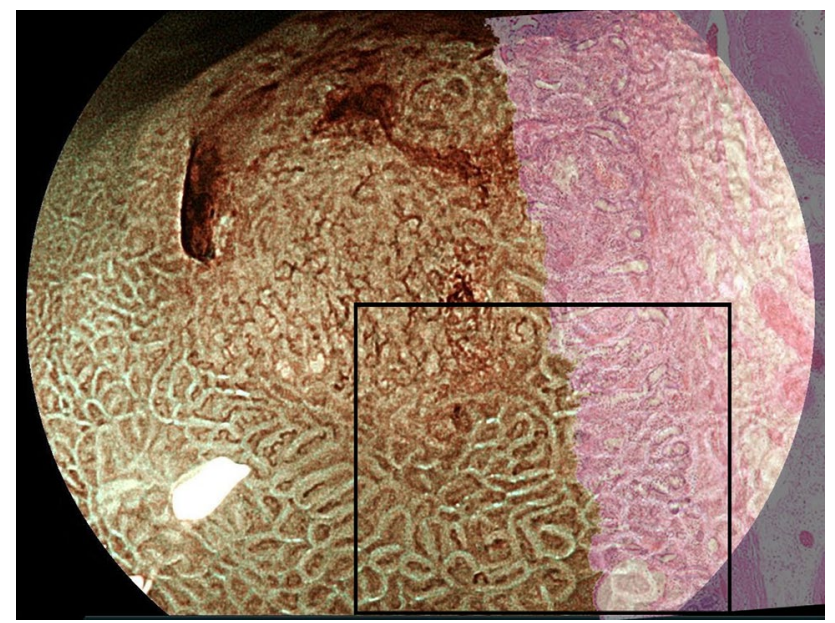

Fig. 6 Step 4: The transparent histological layer laid on the endoscopic image. By setting the opacity of the histological layer to $50 \%$, we could recognize the underlying endoscopic image through the histological layer. The square area outlined in black is shown in Fig. 7

\section{Results}

We successfully adjusted 44 fields (94\%) using the "KOTO Method."

Figures 1, 2, 3, 4, 5, 6, and 7 show a representative case diagnosed histologically as a tubular adenocarcinoma. After fixation in $10 \%$ neutral buffered formalin, the mucosa of the resected tissue was cut vertically at intervals of $2-3 \mathrm{~mm}$ with the deeper tissue being left intact (Fig. 1). After the H\&E sections were prepared and captured in the JPEG format, the photograph of the tissue with mucosal cleavage lines was overlaid with the image of the full-length $\mathrm{H} \& \mathrm{E}$ section (Fig. 2). The overlain image showed rough correspondence between the surface structure and histological vertical image. We then overlaid the more magnified stereoscopic image of the relevant area with the magnified H\&E section image by reference to the rough correspondence and using the same magnification by matching both scale values (Fig. 3). Comparison of the magnified endoscopic image using blue laser imaging-bright mode (BLI-bright: image enhancement mode-A6 and color enhancement mode-C1) obtained by a EG-L590ZW endoscope (FUJIFILM, Tokyo, Japan) with the equivalent stereoscopic image is shown in Fig. 4. This process made possible one-to-one correspondence between the endoscopic and histological images (Fig. 5). By setting the opacity of the histological image layer to $50 \%$ (Fig. 6), we could recognize the underlying surface structure and completely adjust the epithelium of the gland edges to the white zones (Fig. 7a-d).
Fig. 7 Detail of the fitted endoscopic and histological images. a Endoscopic image only. b The endoscopic image was overlaid by the histological layer with the red arrow indicating demarcation of the cancer. c By setting the opacity of the histological layer to $50 \%$, the underlying structure can be recognized allowing complete adjustment of the epithelium of the gland edges to the white zones. $\mathbf{d}$ The correct line in which the H\&E section was made is indicated by the black line; the red arrow indicates demarcation of the cancer
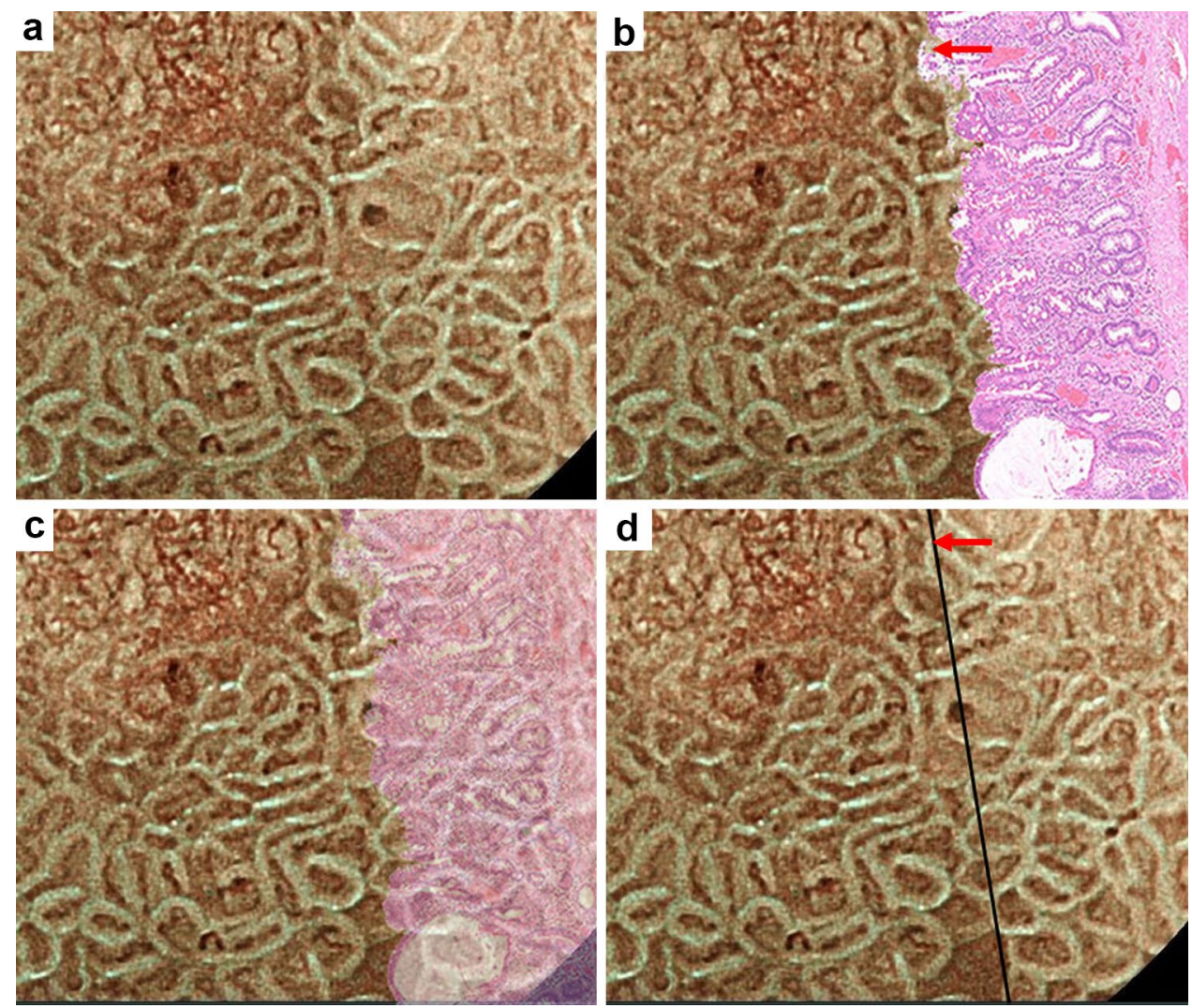


\section{Conclusions}

Recent developments in endoscopy, especially IEE, have provided detailed images of the gastric surface [6-10], which have the potential to attain precise correspondence with histological images. However, this matching has proved difficult because endoscopy shows horizontal images of the surface whereas histology shows vertical structures. Endoscopic magnification was also unclear because of the distortion causing poor estimation of the correct line in the histological section.

We overcame these difficulties by using stereoscopic images to bridge the gap between endoscopic and histological images. Because stereoscopic distortion was considerably less than that of endoscopy, the H\&E section images completely fit the equivalent stereoscopic image. In addition, because mucosal cleavage material pictures were taken, we could demonstrate the correct line on which the $\mathrm{H} \& \mathrm{E}$ section was made by laying the histological image near the cut line of the material on the stereoscopic image and then adjusting both surface structures. By adjusting the scale factor, we could achieve the same magnification for both images. Correspondence of the surface structures between the stereoscopic and endoscopic images allowed adjustment of the histological image to the endoscopic image. To compare the endoscopic surface structures with stereoscopic structures, it is also important that no sections are out of position. Because the submucosal tissue was not completely removed, the mucosal position was maintained, allowing recognition of surface structural continuity. Furthermore, by changing the transparency of histological images we could see both endoscopic and histological images simultaneously and completely fit the histological endoscopic images at a single gland level. However, a limitation of this method is that the images of the H\&E sections did not fit completely with the side of the endoscopic images as a result of the distortion associated with endoscopy. Endoscopic images at an oblique angle also made fitting difficult because the scale ratio changed depending on the distance from the scope. Therefore, it is necessary to take endoscopic images at a front view angle as often as possible.

Histological structures of various gastric cancers are not completely the same even if the cancers are diagnosed as the same histological subtype. Feedback on histological subtypes alone is not sufficient to understand what the endoscopic images demonstrate. To improve endoscopic diagnosis, it is essential to obtain interactive feedback on the one-to-one correspondence between histological structures and endoscopic images. Establishing a histopathologyoriented endoscopic examination may provide more useful information, and consequently the "KOTO Method" is indispensable.

\section{Compliance with ethical standards}

Conflict of interest Y. Naito received collaboration research funding from Fujifilm Medical Co., Ltd., Japan.

Ethical standards This study was approved by the Ethical Review Committee of the KPUM and was carried out in accordance with the Helsinki Declaration of the World Medical Association, with informed consent being obtained from all the patients.

\section{References}

1. Japanese Gastric Cancer Association. Japanese classification of gastric carcinoma, 3rd English edition. Gastric Cancer. 2011;14:101-12.

2. Japanese Gastric Cancer Association. Japanese gastric cancer treatment guidelines 2014 (ver. 4). Gastric Cancer. 2017;20(1):1-19.

3. Gotoda T, Yanagisawa A, Sasako M, Ono H, Nakanishi Y, Shimoda $\mathrm{T}$, et al. Incidence of lymph node metastasis from early gastric cancer: estimation with a large number of cases at two large centers. Gastric Cancer. 2000;3(4):219-25.

4. Hirasawa T, Gotoda T, Miyata S, Kato Y, Shimoda T, Taniguchi $\mathrm{H}$, et al. Incidence of lymph node metastasis and the feasibility of endoscopic resection for undifferentiated-type early gastric cancer. Gastric Cancer. 2009;12(3):148-52.

5. Gotoda T, Jung HY. Endoscopic resection (endoscopic mucosal resection/endoscopic submucosal dissection) for early gastric cancer. Dig Endosc. 2013;25(suppl 1):55-63.

6. Sumiyama K. Past and current trends in endoscopic diagnosis for early stage gastric cancer in Japan. Gastric Cancer. 2017;20(suppl 1):20-7.

7. Yao K. Clinical application of magnifying endoscopy with narrowband imaging in the stomach. Clin Endosc. 2015;48(6):481-90.

8. Dohi O, Yagi N, Majima A, Horii Y, Kitaichi T, Onozawa Y, et al. Diagnostic ability of magnifying endoscopy with blue laser imaging for early gastric cancer: a prospective study. Gastric Cancer. 2017;20(2):297-303.

9. Maki S, Yao K, Nagahama T, Beppu T, Hisabe T, Takaki Y, et al. Magnifying endoscopy with narrow-band imaging is useful in the differential diagnosis between low-grade adenoma and early cancer of superficial elevated gastric lesions. Gastric Cancer. 2013;16(2):140-6.

10. Yu H, Yang AM, Lu XH, Zhou WX, Yao F, Fei GJ, et al. Magnifying narrow-band imaging endoscopy is superior in diagnosis of early gastric cancer. World J Gastroenterol. 2015;21(30):9156-62.

11. Saitoh T, Takamura A, Watanabe G, Sugitani S, Ajioka Y. Effectiveness of magnifying narrow-band imaging endoscopy for differential diagnosis between the high-risk mixed-type and lowrisk simple-type of low-grade, well-differentiated gastric tubular adenocarcinoma. Gastroenterol Res Pract. 2016;2016:3028456.

12. Yagi K, Nozawa Y, Endou S, Nakamura A. Diagnosis of early gastric cancer by magnifying endoscopy with NBI from viewpoint of histological imaging: mucosal patterning in terms of white zone visibility and its relationship to histology. Diagn Ther Endosc. 2012;2012:954809. 